\title{
Peptidoglycan sensing prevents quiescence and promotes quorum-independent growth of uropathogenic Escherichia coli
}

\author{
Eric C. DiBiasio ${ }^{1}$, Hilary J. Ranson ${ }^{2}$, James R. Johnson ${ }^{3}$, David C. Rowley ${ }^{2}$, Paul S. \\ Cohen $^{1}$, Jodi L. Camberg ${ }^{1 *}$ \\ ${ }^{1}$ Department of Cell \& Molecular Biology \\ The University of Rhode Island \\ Kingston, RI 02881 \\ ${ }^{2}$ Department of Biomedical \& Pharmaceutical Sciences \\ The University of Rhode Island \\ Kingston, RI 02881 \\ ${ }^{3}$ Minneapolis Veterans Health Care System \\ Minneapolis, Minnesota, USA; Department of Medicine \\ University of Minnesota \\ Minneapolis, Minnesota, USA \\ *Corresponding author: \\ Jodi L. Camberg \\ 120 Flagg Road, Center for Biotechnology and Life Sciences 479 \\ Department of Cell and Molecular Biology, The University of Rhode Island \\ Kingston, RI, USA 02881 \\ Telephone: (401) 874-4961 \\ Fax: $\quad$ (401) 874-7720 \\ E-mail: $\quad$ cambergj@uri.edu
}

Running Title: Peptidoglycan is a proliferation signal for quiescent UPEC

Keywords: quiescence; dormancy; proliferant; peptidoglycan; quorum; pentapeptide 


\begin{abstract}
The layer of peptidoglycan surrounding bacteria provides structural integrity for the bacterial cell wall. Many organisms, including human cells and diverse bacteria, detect peptidoglycan fragments that are released as bacteria grow. Uropathogenic Escherichia coli (UPEC) strains are the leading cause of human urinary tract infections (UTIs) and many patients experience recurrent infection after successful antibiotic treatment. The source of recurrent infections may be persistent bacterial reservoirs in vivo that are in a quiescent state and, thus, are not susceptible to antibiotics. Here, we show that multiple UPEC strains require a quorum to proliferate in vitro with glucose as the sole carbon source; at low density, the bacteria remain viable but enter a quiescent, non-proliferative state. Of all clinical UPEC isolates tested to date, $35 \%(51 / 145)$ enter this quiescent state, including archetypal strains CFT073 (from classic endemic lineage ST73) and JJ1886 (from recently emerged, multidrug-resistant pandemic lineage ST131). We further show that quorum-dependent UPEC quiescence is prevented and reversed by small molecules, called proliferants, that stimulate growth, such as L-lysine, Lmethionine, and peptidoglycan (PG) stem peptides, including an isolated PG pentapeptide from Staphylococcus aureus. Together, our results indicate that (i) uptake of L-lysine and (ii) PG peptide sensing by UPEC modulate the quorum-regulated decision to proliferate and further demonstrate that PG fragments are important for intra- and interspecies signaling in pathogenic E. coli.
\end{abstract}




\section{Introduction}

At least $80 \%$ of urinary tract infections (UTIs) are caused by uropathogenic Escherichia coli (UPEC) strains, and $27 \%$ of patients with a UTI experience recurrence within 12 months after successful antibiotic treatment $\underline{1-3}$. UPEC appear to enter a quiescent, non-growing state within urothelial transitional cells in the bladder wall that may enable E. coli, long after antibiotic treatment has been terminated, to resume division within bladder urine after apoptotic release from those cells $\underline{4} \underline{5}$.

Recently, we reported the discovery of a new E. coli physiological growth state, common to many UPEC strains $\underline{6}$. That is, the UPEC strain E. coli CFT073, a member of the major UPEC lineage sequence-type (ST) 73, and approximately 80\% of ST73 strains tested grow well in liquid glucose minimal medium, but become quiescent, i.e., fail to grow, but remain viable, on glucose minimal agar plates at a cell density of less than $10^{6} \mathrm{CFU}$ per plate, yet grow well on glucose minimal agar plates seeded with $>10^{6} \mathrm{CFU}$. Moreover, $23 \%$ of randomly selected UPEC strains of diverse STs isolated from community-acquired UTIs also become quiescent on glucose minimal plates seeded with $\leq 10^{6}$ CFU $\underline{6}$. Remarkably, these results indicate that growth of many UPEC strains on glucose minimal agar plates is quorum dependent $\underline{6}$. Alternative sole carbon sources, including acetate, arabinose, fructose, fucose, galactose, gluconate, $N$-acetylglucosamine (NAG), maltose, and mannose also lead to cells entering quiescence at low plating density, whereas glycerol, ribose and xylose promote robust growth $\underline{6}$. We also reported that mini-Tn5 transposon insertions in five central carbon metabolism genes ( $g d h A, g n d, p y k F, s d h A$, and $z w f)$ prevent quiescence, suggesting that metabolic function must remain intact during quiescence even though the cells are nonproliferative. In vitro CFT073 quiescence is prevented when cells are placed near a single 
colony of actively growing CFT073 or E. coli MG1655, a K-12 laboratory strain, suggesting that the actively growing cells release one or more molecules that act as a proliferant. Additionally, we found that L-lysine is a proliferant for quiescent CFT073 cells, but only in combination with either L-methionine or L-tyrosine. Notably, the remaining 17 amino acids play no role in preventing quiescence $\underline{6}$ and CFT073 is not auxotrophic for lysine, methionine or tyrosine. The quorum-dependency of CFT073 proliferation implies that the ability to sense a cell-to-cell signal(s), which may include L-lysine, L-methionine and/or L-tyrosine, underlies the decision by each individual cell to grow and divide or to enter the quiescent state, suggesting that the proliferative/quiescent transition may be controlled by a quorum sensing system $\underline{7}, \underline{8}$.

Here we show that CFT073 quiescent cells are filamentous, that L-lysine must be transported into CFT073 to prevent quiescence, suggesting that L-lysine synthesis is inhibited in quiescent cells, and that peptidoglycan fragments, which are normally secreted by growing E. coli $\underline{9}$, and, more specifically, a peptidoglycan stem pentapeptide, prevent quiescence of two UPEC strains, CFT073, a prototypic ST73 UPEC strain and JJ1886, a pandemic isolate that displays increased sensitivity to proliferants. While peptidoglycan fragments have previously been shown to regulate development of Bacillus subtilis and Mycobacterium tuberculosis, they have not been shown to act as quorum-signaling molecules in UPEC 10-12. These findings demonstrate that population-sensing and metabolite availability modulate the quiescent state of UPEC. In addition, these studies further define an in vitro model system for UPEC quiescence that will enable closer examination of quiescent UPEC physiology and may lead to new approaches to prevent recurrent UTI. 


\section{RESULTS}

\section{Quorum-dependent growth of UPEC and prevention of quiescence on glucose minimal}

agar plates by secreted signals. UPEC strain CFT073 enters a quiescent state when plated at low cell density $\left(10^{4}\right.$ cells per plate) on glucose minimal agar, yet grows robustly when plated at high cell density ( $10^{8}$ cells per plate), demonstrating that growth of CFT073 on glucose minimal agar plates is quorum-dependent (Fig. S1A) $\underline{6}$. As reported previously, quiescent CFT073 cells can be resuscitated by challenge with specific external stimuli, called proliferants, including additions of $5 \mu \mathrm{l}$ of a solution containing L-lysine (1 mM) and Lmethionine $(1 \mathrm{mM}), 5 \mu \mathrm{l}$ of human urine, or a single colony of actively growing $E$. coli spp. (CFT073 or MG1655), added to the plate $\underline{6}$. In a soft agar overlay containing $10^{4}$ quiescent CFT073 cells, we tested known proliferants and observed the development of a zone of actively growing colonies surrounding the site of challenge for all proliferants tested over $24 \mathrm{~h}$ (Fig. 1A). Without challenge, cells failed to grow throughout the course of the experiment and remained quiescent. We also tested additional clinical isolates and found that quorumdependent growth on glucose minimal agar is common among the ST131 UPEC strains in this study (8/33) (Table 1) and among other isolates in a previous study $\underline{6}$; however, we discovered that the number of cells constituting a quorum varies among strains. For example, the ST131 strain JJ1886 was proliferative on glucose minimal agar at a plating density greater than $10^{3}$ CFU, but quiescent at or below $10^{3} \mathrm{CFU}$ (Table 1). Of the 35 strains tested in this study, 10 were reversibly quiescent on glucose minimal agar, and three of those strains were quiescent at or below a plating density of $10^{3} \mathrm{CFU}$, but non-quiescent at $10^{5} \mathrm{CFU}$ (Table 1). These results show that sensitivity for quorum detection varies by strain, i.e., is strain-specific. 
Next, we monitored the zone of growth of CFT073 cells as they exited quiescence over $72 \mathrm{~h}$. First, we poured soft agar plates containing quiescent CFT073 cells seeded at $10^{4} \mathrm{CFU}$ per plate, placed an actively growing colony of CFT073 onto the surface of each plate by toothpick and measured the zones of growth around the sites of inoculation at 24,48 and $72 \mathrm{~h}$. We observed that the zone of growth expands in diameter until the entire plate is covered by a lawn of actively growing cells (Fig. 1B and 1C). There are two potential scenarios to explain this result. First, the proliferant(s) that stimulates growth of quiescent cells is continuously released from the toothpicked colony throughout the experiment and induces growth of distal quiescent cells as it diffuses across the plate. Alternatively, the proliferant(s) that stimulates growth of quiescent cells is released from the toothpicked colony but, upon release of the signal, quiescent cells, once stimulated to proliferate, secrete their own signal, thereby stimulating adjacent quiescent cells to grow. To distinguish between these possibilities, glucose minimal agar plates were seeded with $10^{4}$ quiescent CFT073 cells from a stationary phase liquid glucose minimal medium culture, and a single CFT073 colony was toothpicked onto the center of each plate. The plates were then incubated for $24 \mathrm{~h}$. During that time, a small zone of growth formed on each plate around the central toothpicked colony (Fig. 1B, 1C and 1D). After the zone had formed at $24 \mathrm{~h}$, the toothpicked colony was removed to stop the colony from secreting additional signal(s). Even after removal of the toothpicked colony from the plate, the zone of growth continued to expand, unabated, for an additional $48 \mathrm{~h}$ and at a similar rate as the control plate, where the colony had been left intact (Fig. 1C and 1D). These results suggest that the proliferation-promoting signal propagates across the plate as CFT073 cells exit quiescence. 
These results further show that quiescent CFT073 cells at the edge of the plate remain viable and can be stimulated to proliferate for up to $72 \mathrm{~h}$.

\section{Quiescent CFT073 cells are filamentous on glucose minimal agar plates. During}

uropathogenic infection, bacterial cells from quiescent intracellular reservoirs have several different morphologies, including rods, spheres, and filaments 13-15. To determine if quiescent cells in our in vitro quiescence system also display morphological features that are distinct from actively growing cells, we compared the cell morphologies of CFT073 cells under several culture conditions, including LB agar and glucose minimal agar supplemented with L-lysine $(1 \mathrm{mM})$ and L-methionine $(1 \mathrm{mM})$, where indicated (Fig. 2A). After $24 \mathrm{~h}$ on LB agar or on glucose minimal agar supplemented with L-lysine and L-methionine, colonies were apparent and individual cells collected appeared as short rods with average lengths of $1.72 \pm 0.59 \mu \mathrm{m}$ $(\mathrm{n}=200)$ and $2.33 \pm 0.80 \mu \mathrm{m}(\mathrm{n}=200)$, respectively (Fig. 2A and $2 \mathrm{~B})$. However, after $24 \mathrm{~h}$ on glucose minimal agar plates, no colonies were detected and quiescent cells harvested from the plate had a broad length distribution with a mean length of $7.17 \pm 4.34 \mu \mathrm{m}(\mathrm{n}=200)$, which is $300 \%$ longer than cells grown on glucose minimal agar supplemented with lysine and methionine (Fig. 2A and 2B). Many quiescent cells were longer than $10 \mu \mathrm{m}$ and $16.5 \%$ of the cells appeared to contain one or more incomplete septa (Fig. 2A and 2B) (Fig. S1B). To determine if cell length changes upon extended incubation, we also measured cell length at 0 $\mathrm{h}$ and $48 \mathrm{~h}$ after entry into quiescence. At $0 \mathrm{~h}$, cells harvested from a stationary phase culture grown in liquid glucose minimal medium were short rods with an average cell length of 2.14 $\pm 0.58 \mu \mathrm{m}(\mathrm{n}=200)$. After $48 \mathrm{~h}$ on glucose minimal agar plates, quiescent cells were filamentous with an average cell length of $6.84 \pm 3.76 \mu \mathrm{m}(\mathrm{n}=72)$, which is similar to the 
average length at $24 \mathrm{~h}$ (Fig. S1B and S1C). These results suggest that quiescent CFT073 cells elongate during the first $24 \mathrm{~h}$ on the plate but fail to complete division.

\section{Lysine import is necessary for lysine to prevent quiescence, but an additional signal that}

induces proliferation is released from actively growing cells. L-lysine is a proliferant for quiescent CFT073 cells on glucose minimal agar containing L-methionine. To determine whether L-lysine must be imported into CFT073 cells to prevent quiescence, we constructed a CFT073 lys $P$ insertion-deletion mutant by $\lambda$-Red recombination (Table S1). Of the three known lysine importers in E. coli, LysP is the major importer and a member of the amino acid, polyamine and organocation (APC) family of transporters $\underline{16} \underline{17}$. CFT073 $\Delta l y s P$ cells grew well overnight in liquid glucose minimal medium, similar to the wild type CFT073 strain (Fig. S2A) and exhibited quiescence on glucose minimal agar at low cell density ( $10^{4}$ cells per plate) (Fig. 3); yet, it grew robustly when plated at high density $\left(10^{8}\right.$ cells per plate). Unlike wildtype CFT073, when $5 \mu 1$ of a solution of L-lysine $(1 \mathrm{mM})$ and L-methionine $(1 \mathrm{mM})$ was added to quiescent CFT073 $\Delta l y s P$ cells plated at $10^{4} \mathrm{CFU}$, no colony growth was observed after $24 \mathrm{~h}$ of incubation suggesting that LysP-dependent transport is critical for lysine to stimulate proliferation. This suggests that lysine biosynthesis is inhibited in quiescent cells. CFT073 $\Delta l y s P$ was complemented with a plasmid containing lys $P$ (pLysP), which restored the ability of the strain to proliferate upon addition of L-lysine in the presence of L-methionine at low cell density (Fig. 3). Although quiescent CFT073 $\Delta l y s P$ cells failed to respond to L-lysine, they proliferated after a colony of actively growing CFT073 cells was added to the plate (Fig. 3). In addition, CFT073 $\Delta l y s P$ quiescent cells also proliferated when $5 \mu l$ of human urine was added to the plate (Fig. 3). These results suggest that import of L-lysine is required for L-lysine 
to stimulate proliferation and override the absence of the quorum. Moreover, one or more Llysine-independent proliferants that reverse quiescence are secreted from bacterial colonies and are present in urine.

$\operatorname{ArgP}$ is a transcriptional regulator of $\operatorname{lys}_{\mathrm{P}} \underline{16} \underline{18}$. In the absence of lysine, ArgP positively regulates transcription of $l_{y s} P$, as well as several $E$. coli genes involved in lysine and peptidoglycan biosynthesis, including $d a p B$ (dihydropicolinate reductase), dapD (tetrahydrodipicolinate succinylase), $\operatorname{lys}_{s}$ (diaminopimelate decarboxylase) and lys $C$ (aspartokinase III) $\underline{16} \underline{18-20}$. To test whether ArgP plays a regulatory role in quorum-dependent growth or quiescence, we deleted $\arg P$. Like wild type CFT073, CFT073 $\triangle \arg P$ cells grew well overnight in liquid glucose minimal medium (Fig. S2A) and were quiescent at low cell density $\left(10^{4}\right.$ cells per plate) on glucose minimal agar (Fig. 3) indicating that ArgP is not essential for inducing quiescence at low density (Fig. 3). Similar to the result with CFT073 $\Delta l y s P$ cells, quiescent CFT073 $\triangle \arg P$ cells failed to respond to the addition of L-lysine with L-methionine after $24 \mathrm{~h}$ of incubation, but the cells proliferated in response to a colony of CFT073 toothpicked to the center of a glucose minimal agar plate seeded with $10^{4} \mathrm{CFU}$ CFT073 $\triangle \arg P$ (Fig. 3). Complementation of CFT073 $\triangle \arg P$ with the wildtype CFT073 $\arg P$ gene on a plasmid ( $\mathrm{pArgP}$ ) restored the ability of the strain to respond to L-lysine in the presence of L-methionine at low cell density (Fig. 3). Since LysP expression has been reported to be repressed 35 -fold in E. coli $\arg P$ mutants 20 , this result supports the conclusion that external L-lysine must be transported into CFT073 via LysP in order to prevent quiescence and confirms that expression of LysP is regulated by ArgP. Moreover, these results also show 
that there is another molecule secreted by actively growing cells that stimulates proliferation of quiescent CFT073 cells.

To further understand why the population that defines a quorum varies between strains, we investigated the ST131 clinical isolate JJ1886, which exhibits quiescence on glucose minimal agar at or below a plating density of $10^{3} \mathrm{CFU}$ but grows robustly at $10^{5} \mathrm{CFU}$ and in liquid glucose minimal medium (Fig. 4A and S2B). We observed that $5 \mu$ of a solution of L-lysine $(1 \mathrm{mM})$ alone is sufficient to promote growth and prevent quiescence of JJ1886 plated at low density on glucose minimal agar $\left(10^{3} \mathrm{CFU}\right.$ per plate), although growth is more efficient in the presence of L-methionine $(1 \mathrm{mM})$, and L-methionine $(1 \mathrm{mM})$ alone does not stimulate growth (Fig. 4B). Since quiescent JJ1886 cells on glucose minimal agar proliferate in the presence of L-lysine alone, we titrated the L-lysine concentration to determine the threshold for stimulation of growth and compared JJ1886 to CFT073. We determined that a low concentration of Llysine $(5 \mu \mathrm{l}$ of $0.1 \mathrm{mM})$ is sufficient to promote proliferation of quiescent JJ1886 cells. In contrast, quiescent CFT073 cells only proliferate at a higher concentration of L-lysine ( $5 \mu 1$ of $1 \mathrm{mM}$ ) and only in the presence of L-methionine. These results suggest that although JJ1886 behaves phenotypically similar to CFT073 with respect to quiescence, it is more sensitive to detecting or responding to an external signal(s) that prevents quiescence. We then tested if quiescent JJ1886 also responds to the other known CFT073 proliferants, including $5 \mu$ l of urine or a colony of actively growing $E$. coli cells, and we observed that both were effective for stimulating proliferation of quiescent JJ1886 cells (Fig. 4B). 
Since L-lysine stimulates proliferation of both JJ1886 and CFT073, we tested $5 \mu$ l of D-lysine $(1 \mathrm{mM})$ and $5 \mu 1$ of the biosynthetic lysine precursor diaminopimelic acid (Dap) (1 mM) for stimulating proliferation of quiescent cells. Both failed to stimulate growth of quiescent CFT073 and JJ1886 (Fig. S3A and S3B).

\section{Peptidoglycan fragments prevent UPEC quiescence.}

Next, we considered peptidoglycan (PG) as a potential signal that would communicate a quorum since peptidoglycan fragments, including di-, tri-, and tetrapeptides, are normally secreted by actively growing bacterial cells including E. coli $\underline{9}, \underline{11}, \underline{21}, \underline{22}$. We tested if peptidoglycan fragments from $E$. coli could prevent quiescence and stimulate proliferation of CFT073 and JJ1886. Peptidoglycan was digested with mutanolysin, a mixture of two Nacetylmuramidases and an N-acetylmuramyl-L-alanine amidase, to release a heterogeneous mixture of peptidoglycan fragments, including various muropeptides and stem peptides (Fig. 5A). The addition of the total mixture of digested peptidoglycan fragments from E. coli induced proliferation of quiescent JJ1886 cells, plated at $10^{3} \mathrm{CFU}$ per plate (Fig. 5A), and quiescent CFT073 cells, plated at $10^{4} \mathrm{CFU}$ per plate (Fig. S4A). Titration of the amount of total peptidoglycan added to the plate revealed dose-dependent detection of fragments leading to JJ1886 colony growth in a zone around the site of addition (Fig. S4B). Digested peptidoglycan from Bacillus subtilis and Staphylococcus aureus also stimulated proliferation of quiescent JJ1886 and CFT073 cells (Fig. S4C and S4D). Furthermore, peptidoglycan fragments were also effective for stimulating quiescent CFT073 $\Delta l y s P$ cells to proliferate, indicating that the peptidoglycan response is independent of lysine import (Fig. S5A). Interestingly, these results show that quiescent $E$. coli cells respond to peptidoglycan fragments 
from various bacterial species, which can differ in the identity of the amino acid at the third position of the stem peptide, usually meso-Dap (i.e., E. coli) or L-lysine (i.e., S. aureus) $\underline{11}, \underline{23}, \underline{24}$

\section{A peptidoglycan stem pentapeptide acts as a signal to promote proliferation of quiescent}

\section{JJ1886 and CFT073 cells}

To identify which fragment(s) of digested peptidoglycan stimulates proliferation of quiescent cells, we performed high performance liquid chromatography-mass spectrometry (HPLC-MS) on E. coli peptidoglycan fragment mixtures (Fig. 5A) and tested fractions across the elution for the ability to promote proliferation of quiescent JJ1886 cells. We found that early fractions collected, with retention times up to 10 minutes were sufficient to stimulate proliferation of quiescent JJ1886 cells (Fig. S5B). Tandem mass spectrometry confirmed that these fractions contain low molecular weight peptidoglycan fragments, consistent with stem peptides, such as the tri- and tetrapeptides.

To further understand the molecular determinants of PG peptides that stimulate proliferation of quiescent cells, we compared several highly pure stem peptide fragments of varying amino acid compositions and lengths. We observed that a tripeptide, representing the minimal unit of an E. coli peptidoglycan stem peptide that could be generated from amidase and carboxypeptidase activities in the $E$. coli periplasm (L-Ala-D-Glu- $m$ Dap), failed to stimulate proliferation of quiescent JJ1886 cells (Fig. 5B). However, a peptidoglycan pentapeptide, LAla-D-Glu-L-Lys-D-Ala-D-Ala (A-E-K-A-A), corresponding to the stem pentapeptide present in S. aureus peptidoglycan, stimulated proliferation of quiescent JJ1886 (Fig. 5C) and CFT073 
(Fig. S5A) cells. We also tested a smaller fragment of the pentapeptide, acetyl-L-Lys-D-AlaD-Ala (K-A-A) but did not observe stimulatory activity (Fig. S6A). Fragments including the disaccharide moiety alone, containing NAG and N-acetyl muramic acid (NAM) (NAG-NAM) (Fig. 5D), and higher molecular weight molecules with the attached stem peptide (i.e., disaccharide tetrapeptide) (Fig. 5E), in various states of crosslinking, eluted later, after 10 min, and had no proliferant activity (Fig. S7). NAG-NAG, NAG and NAM were tested, but none of these sugars stimulated proliferation of quiescent cells (Fig. S6B). These results further suggest that a stem peptide functions as a signal to promote proliferation of quiescent cells.

Finally, to further clarify if a stimulatory peptide, such as the pentapeptide, exerts its function without entering the cytoplasm or if it is imported into a quiescent cell and enters the peptidoglycan recycling pathway, we constructed JJ1886 strains deleted for the oligopeptide transporter operon, $o p p A B C D F \underline{25-29}$, as well as the peptidoglycan tripeptide-specific delivery

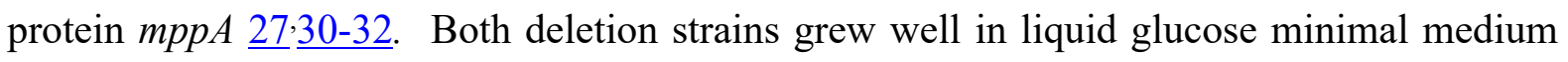
and became quiescent when plated at $10^{3} \mathrm{CFU}$ per plate, similar to the parental strain JJ1886; however, both strains were stimulated to proliferate upon addition of the pentapeptide to the plate (Fig. S8), indicating that it is not necessary for the pentapeptide to enter the cytoplasm via the Opp transporter to prevent quiescence and therefore it may act as a signal to prevent and reverse quiescence.

\section{DISCUSSION}

Here, we show that two virulent UPEC strains, CFT073 and JJ1886, enter a quiescent state when plated on glucose minimal agar that is prevented and reversed by proliferants, including 
a pentapeptide derived from PG, and L-lysine. Quiescence is a unique physiological state recently described for $E$. coli that is common among UPEC strains (51/145 isolates tested) and regulated by the presence or absence of a quorum. Several notable features of the E. coli quiescent phenotype are described here. First, the quiescent state of UPEC appears to be a bona fide developmental state that is adopted by an entire population of cells under a given growth condition. This is in contrast to persistence, which occurs in a small percentage of cells $\underline{33-37}$. Second, adoption of a non-proliferative state would require that many physiological systems would be slowed or inhibited, including DNA replication, transcription, translation, cell division and peptidoglycan synthesis. Accordingly, we observed that although quiescent cells adopt a filamentous morphology, they fail to divide or successfully proliferate upon extended incubation (Fig. S1B). Furthermore, as described previously, mutations in five central carbon metabolism genes ( $g d h A, g n d, p y k F, s d h A$, and $z w f)$ prevent quiescence and promote growth, indicating that specific metabolic pathways must be functional to maintain the quiescent state 6. Finally, different strains respond to known proliferants with varying sensitivities, suggesting that either sensitivity of detection or regulatory control over the physiological systems varies among strains.

Peptidoglycan muropeptide fragments are known to resuscitate dormant Mycobacterium smegmatis $\underline{38}$ and to signal Bacillus subtilis endospores to germinate $\underline{12}$. Here in E. coli, peptidoglycan fragments not only cause cells to reenter the cell cycle, but they may also act as a signal to communicate the presence of a quorum, although they do not appear to require uptake by the Opp transport system. Previously, it was shown that an oppA mutant is far less infective in a mouse UTI model than the wildtype $\underline{39}$, and it was suggested that small oligopeptides, present in high concentration in urine 39 , are imported and serve as major 
nutrients for CFT073 growth in urine $\underline{39}$. Here, the pentapeptide does not enter the quiescent cells via Opp. Therefore, either the pentapeptide is imported by a second, presently unknown, oligopeptide permease or the pentapeptide acts as a signal in the periplasm and is recognized by a membrane receptor or signal transduction system that, when activated, allows cell proliferation. The serine/threonine kinase PknB is a widely conserved peptidoglycan binding protein that is important for germination of spores in B. subtilis $\underline{12}$; however, no similar proteins have been found thus far in E. coli.

The cause(s) of recurrent UTIs are complex $\underline{40}$, however, it appears that UPEC can bind to, enter, and replicate within superficial facet cells in the mouse and human bladder epithelium, resulting in intracellular biofilm-like communities (IBCs) $\underline{40} \underline{41}$. IBCs escape from infected superficial facet cells within hours of development and several cycles of IBC formation occur, but each successive round is associated with a reduced replication rate and smaller IBCs $\underline{15}$. The infected superficial facet cells exfoliate, exposing underlying transitional epithelial cells that become infected with IBC-derived UPEC progeny. Upon infection of transitional cells, small numbers of UPEC appear to enter a latent or quiescent intracellular state in endosomal vesicles ( 2 to 12 cells/vesicle), establishing what have been termed quiescent intracellular reservoirs (QIRs) $\underline{42}$ resistant to antibiotic treatment. Our results from in vitro quiescence assays with UPEC described here suggest that upon release from endosomal vesicles, UPEC may sense proliferants and thus be stimulated to reenter the cell cycle.

\section{MATERIALS AND METHODS}

\section{Bacterial strains, plasmids and growth conditions}


E. coli strains and plasmids used in this study are listed in Table 1. Clinical UPEC isolates were obtained from the collections of J. Johnson (29 strains) and E. Sokurenko (5 strains) (Table 2). LB agar (Lennox) was used for routine cultivation. Amino acids and M9 minimal medium and agar with glucose $(0.2 \%$ or $0.4 \%$, where indicated $)$ were prepared as described by 6. Gene deletions were constructed by $\lambda$-Red recombination as described in Datsenko and Wanner and confirmed by sequencing 43 . Culture density was monitored by growing cells in liquid $0.2 \%$ glucose M9 minimal medium and measuring $\mathrm{OD}_{600}$ at the indicated times.

\section{Complementation}

Plasmids for complementation of both CFT073 $\Delta l y s P$ and CFT073 $\Delta \arg P$ gene deletions were constructed using expression vector pBAD24. Both genes were amplified from CFT073 by whole colony PCR. The resulting PCR products were digested with EcoRI and HindIII. Digested PCR products were ligated into EcoRI/HindIII-digested pBad24. Both pLysP and pArgP plasmid constructs were verified by sequencing and then transformed into either CFT073 $\triangle l y s P$ or CFT073 $\triangle \arg P$ deletion strains by electroporation. To test for complementation, transformants were grown overnight in $0.2 \%$ glucose M9 minimal medium with ampicillin $\left(50 \mu \mathrm{g} \mathrm{ml}^{-1}\right)$ and assayed for quiescence on glucose minimal agar supplemented with arabinose $(0.1 \%)$, where indicated.

\section{Quiescence assay}

Quiescence assay was performed as described previously $\underline{6}$. CFT073 and JJ1886 wild type and mutant strains were grown on LB agar plates overnight at $37^{\circ} \mathrm{C}$. An isolated colony was used to inoculate $5 \mathrm{ml}$ of $0.2 \%$ glucose M9 minimal media, incubated at $37{ }^{\circ} \mathrm{C}$ with shaking 
overnight, and then diluted to between $10^{3}$ to $10^{8} \mathrm{CFU}$, as indicated, in $3 \mathrm{ml} 0.2 \%$ glucose M9 minimal medium containing $0.75 \%$ Difco noble agar held at $45^{\circ} \mathrm{C}$. Inoculated soft agar was immediately poured onto plates containing a $12 \mathrm{ml}$ layer of $0.2 \%$ glucose $\mathrm{M} 9$ minimal medium with $1.5 \%$ noble agar or directly into an $8.5 \mathrm{~cm}^{2}$ polystyrene culture dish. Proliferants, including actively growing cells, amino acids, Dap (2,6- diaminopimelic acid), sterile human urine and peptidoglycan, were added as indicated and plates were incubated at $37^{\circ} \mathrm{C}$ between 24 and $72 \mathrm{~h}$ and then imaged.

\section{Microscopy}

Overnight cultures of CFT073 were grown to stationary phase in $0.2 \%$ glucose M9 minimal media. Five $\mu 1$ of a 3-log diluted culture was spotted onto LB agar or $0.2 \%$ glucose M9 minimal medium with $1.5 \%$ noble agar and, where indicated, supplemented with L-lysine $(1 \mathrm{mM})$ and L-methionine $(1 \mathrm{mM})$, then allowed to dry for $1 \mathrm{~h}$ at room temperature. Plates were incubated at $37^{\circ} \mathrm{C}$ for 24 or $48 \mathrm{~h}$. Cells were harvested onto a glass cover slip, resuspended in phosphatebuffered saline (PBS) with ethylenediaminetetraacetic acid (EDTA) (1 mM) and applied to an agar gel pad containing $0.2 \%$ glucose M9 minimal with $0.5 \%$ noble agar. Cells were visualized by differential interference contrast (DIC) microscopy using a Zeiss LSM 700, and images were captured on an AxioCam HRc high-resolution camera with ZEN 2012 software. Images were processed using Adobe Photoshop CC and analyzed using NIH ImageJ software.

\section{Peptidoglycan fragment separation}

E. coli CFT073 was inoculated in $10 \mathrm{ml}$ of M9 minimal medium containing $0.4 \%$ glucose and incubated at $37^{\circ} \mathrm{C}$ shaking (200 rpm) for $24 \mathrm{~h}$. Cultures were subsequently inoculated into one 
liter of M9 minimal media containing $0.4 \%$ glucose and incubated for 24 h shaking (175 rpm). Methods used for peptidoglycan isolation were adapted from Desmarais et al., $2014 \underline{44}$. Cells were harvested at 5,000 $\mathrm{x}$ for 10 minutes at room temperature and pellets were resuspended in $3 \mathrm{ml}$ of M9 minimal medium. Cell suspensions were transferred into $6 \mathrm{ml}$ of $6 \%$ sodium dodecyl sulfate (SDS) and boiled for $3 \mathrm{~h}$ with stirring. After $3 \mathrm{~h}$, heat was discontinued, and cell suspensions were stirred overnight. Cell suspensions were centrifuged at 262,000 x $g$ for 30 minutes, pellets were resuspended in liquid chromatography mass spectrometry (LCMS) grade water and further centrifugation and wash steps occurred until no SDS remained. Final pellet was resuspended in $900 \mu \mathrm{l}$ of $10 \mathrm{mM}$ Tris- $\mathrm{HCl}$ with $0.06 \% \mathrm{w} / \mathrm{v} \mathrm{NaCl}$ and $100 \mu \mathrm{g}$ of Pronase E was added to the cell suspension and then incubated for $2 \mathrm{~h}$ at $60{ }^{\circ} \mathrm{C}$. After incubation, $200 \mu \mathrm{l}$ of $6 \%$ SDS was added and the mixture was boiled at $100{ }^{\circ} \mathrm{C}$ for $30 \mathrm{~min}$. Samples were centrifuged at 262,000 $\mathrm{x} g$ for 30 min washed 4 times with LCMS grade water. The final pellet was resuspended in $200 \mu \mathrm{l}$ of PBS and incubated with $200 \mathrm{U}$ of mutanolysin (Sigma-Aldrich) at $37^{\circ} \mathrm{C}$ overnight. Samples were centrifuged at $16,000 \times \mathrm{g}$ for $10 \mathrm{~min}$ to remove cellular debris, peptidoglycan supernatant was collected for further testing. Mutanolysin was removed by ultrafiltration using a polyethersulfone filter with a $3 \mathrm{kDa}$ molecular weight cut-off.

Analytical high-performance liquid chromatography (HPLC) was used to purify the peptidoglycan extract. HPLC experiments were performed on a Shimadzu prominence-I LC2030C equipped with a PDA detector model LC-2030/2040, pump LC-2030, and autosampler LC-2030. Preparative HPLC experiments were completed with a Waters XBridge Analytical $\mathrm{C} 185 \mu \mathrm{m}, 4.6 \times 250 \mathrm{~mm}$ column at $30{ }^{\circ} \mathrm{C}$ under a solvent gradient of $10-100 \% \mathrm{~B}(5 \mathrm{mM}$ 
ammonium acetate with $15 \%$ acetonitrile $\mathrm{pH} 4.89$ ) run over 16 minutes against solvent $\mathrm{A}$ ( $5 \mathrm{mM}$ ammonium acetate $\mathrm{pH} 4.37)$. Solvent $\mathrm{B}$ was held at $10 \%$ for 4 minutes prior to gradient beginning and 100\% B was held for a subsequent 4 minutes. Individual peaks were collected according to $205 \mathrm{~nm}$ absorbance values. Initial chemical characterization was carried out by liquid chromatography mass spectrometry (LCMS) on a Thermo Fisher ISQ EC coupled to a Shimadzu Prominence-i LC-2030 system. Chemical separation utilized the same chemical gradient as previously with a reduced flow rate of $0.6 \mathrm{ml} \mathrm{min}{ }^{-1}$. Chemical characterization of pure compounds was undertaken using electrospray ionization mass spectrometry (ESIMS) using an AB Sciex TripleTOF 4600 spectrometer and LC-MSMS experiments.

LC-MSMS methods were performed on a AB Sciex TripleTOF 5600 spectrometer coupled to a Waters H class UPLC. LC methods mirrored that of initial screening with an adjusted flow rate of $0.5 \mathrm{~mL} / \mathrm{min}$ on an XBridge Analytical C18 $5 \mu \mathrm{m}, 4.6 \times 250 \mathrm{~mm}$ column at room temperature. SWATH acquisition was performed in positive ionization mode. The method specific parameters were as follows: gas 1 (GS1) 55 psi, gas 2 (GS2) 60 psi, curtain gas (CUR) 25 psi. The source-specific parameters were: temperature (TEM) $500^{\circ} \mathrm{C}$, ion spray voltage floating (ISVF) $5500 \mathrm{~V}$, declustering potential (DP): 100, collision energy (CE) 10, collision energy spread (CES) 15. An initial TOF scan was collected from $m / z 100$ to 1500 and SWATH data was acquired ( $m / z$ 200-1100) over 36 SWATH windows per cycles with a window size of $m / z 25$ and a $m / z 1$ overlap between windows.

Peptidoglycan-derived stem peptide fragments including 'A-E-K-A-A' pentapeptide (L-AlaD-Glu-L-Lys-D-Ala-D-Ala (Sigma-Aldrich), 'K-A-A' tripeptide (acetyl-L-Lys-D-Ala-D-Ala) 
bioRxiv preprint doi: https://doi.org/10.1101/830877; this version posted November 4, 2019. The copyright holder for this preprint (which was not certified by peer review) is the author/funder, who has granted bioRxiv a license to display the preprint in perpetuity. It is made available under aCC-BY-NC-ND 4.0 International license.

(Sigma-Aldrich) and 'A-E-Dap' tripeptide (L-Ala-D-Glu- $m$ Dap) (AnaSpec), were each tested at $5 \mu \mathrm{l}(2 \mathrm{mM})$ against strains JJ1886, JJ1886 $\triangle$ opp $A B C D F$ and JJ1886 $\triangle m p p A\left(10^{3}\right)$ grown on M9 minimal agar with $0.2 \%$ glucose for $24 \mathrm{~h}$ at $37^{\circ} \mathrm{C}$. 


\section{Supplemental information is provided as a separate file.}

\section{Table S1}

Figure S1, S2, S3, S4, S5, S6, S7, S8

\section{Funding Information}

Research reported in this publication was supported by the National Institute of General Medical Sciences of the National Institutes of Health under Award Number R01GM118927 to J. Camberg. The content is solely the responsibility of the authors and does not necessarily represent the official views of the National Institutes of Health.

\section{ACKNOWLEDGEMENTS}

We thank Cathy Trebino, Evelyn Siler, Josiah Morrison, Negar Rahmani, and Colby Ferreira for critical reading of the manuscript, Rebecca Dickinson for technical assistance, Barry Wanner for pKD267, Tyrrell Conway for helpful discussions, Rod Welch for CFT073 comparison strain, Evgeni Sokurenko for UPEC strains and plasmids, and Janet Atoyan for microscopy and sequencing assistance. Microscopy and sequencing were performed at the Rhode Island Genomics and Sequencing Center, which is supported in part by the National Science Foundation (MRI Grant No. DBI-0215393 and EPSCoR Grant No. 0554548 \& EPS1004057), the US Department of Agriculture (Grant Nos. 2002-34438-12688, 2003-3443813111, and 2008-34438-19246) and the University of Rhode Island. 


\section{References}

1 Foxman, B. Recurring urinary tract infection: incidence and risk factors. American Journal of Public Health 80, 331-333 (1990).

2 Foxman, B. Epidemiology of urinary tract infections: incidence, morbidity, and economic costs. The American Journal of Medicine 113 Suppl 1A, 5S-13S (2002).

3 Brauner, A., Jacobson, S. H. \& Kuhn, I. Urinary Escherichia coli causing recurrent infections--a prospective follow-up of biochemical phenotypes. Clinical Nephrology 38, 318-323 (1992).

4 Kerrn, M. B., Struve, C., Blom, J., Frimodt-Moller, N. \& Krogfelt, K. A. Intracellular persistence of Escherichia coli in urinary bladders from mecillinam-treated mice. The Journal of Antimicrobial Chemotherapy 55, 383-386, doi:10.1093/jac/dki002 (2005).

5 Blango, M. G. \& Mulvey, M. A. Persistence of uropathogenic Escherichia coli in the face of multiple antibiotics. Antimicrobial Agents and Chemotherapy 54, 1855-1863, doi:10.1128/AAC.00014-10 (2010).

6 Leatham-Jensen, M. P. et al. Uropathogenic Escherichia coli Metabolite-Dependent Quiescence and Persistence May Explain Antibiotic Tolerance during Urinary Tract Infection. mSphere 1, 1-22, doi:DOI: 10.1128/mSphere.00055-15 (2016).

7 Waters, C. M. \& Bassler, B. L. Quorum sensing: cell-to-cell communication in bacteria. Annual Review of Cell and Developmental Biology 21, 319-346, doi:10.1146/annurev.cellbio.21.012704.131001 (2005).

8 Whiteley, M., Diggle, S. P. \& Greenberg, E. P. Progress in and promise of bacterial quorum sensing research. Nature 551, 313-320, doi:10.1038/nature24624 (2017). 
9 Goodell, E. W. \& Schwarz, U. Release of cell wall peptides into culture medium by exponentially growing Escherichia coli. Journal of Bacteriology 162, 391-397 (1985).

10 Peddireddy, V., Doddam, S. N. \& Ahmed, N. Mycobacterial Dormancy Systems and Host Responses in Tuberculosis. Front Immunol 8, 84, doi:10.3389/fimmu.2017.00084 (2017).

11 Irazoki, O., Hernandez, S. B. \& Cava, F. Peptidoglycan Muropeptides: Release, Perception, and Functions as Signaling Molecules. Frontiers in Microbiology 10, 500, doi:10.3389/fmicb.2019.00500 (2019).

12 Shah, I. M., Laaberki, M. H., Popham, D. L. \& Dworkin, J. A eukaryotic-like Ser/Thr kinase signals bacteria to exit dormancy in response to peptidoglycan fragments. Cell 135, 486496, doi:10.1016/j.cell.2008.08.039 (2008).

13 Mulvey, M. A., Schilling, J. D. \& Hultgren, S. J. Establishment of a persistent Escherichia coli reservoir during the acute phase of a bladder infection. Infection and Immunity 69 , 4572-4579, doi:10.1128/IAI.69.7.4572-4579.2001 (2001).

14 Anderson, G. G. et al. Intracellular bacterial biofilm-like pods in urinary tract infections. Science 301, 105-107, doi:10.1126/science.1084550 (2003).

15 Justice, S. S. et al. Differentiation and developmental pathways of uropathogenic Escherichia coli in urinary tract pathogenesis. Proceedings of the National Academy of Sciences of the United States of America 101, 1333-1338, doi:10.1073/pnas.0308125100 (2004).

16 Ruiz, J., Haneburger, I. \& Jung, K. Identification of ArgP and Lrp as transcriptional regulators of $y_{s} P$, the gene encoding the specific lysine permease of Escherichia coli. Journal of Bacteriology 193, 2536-2548, doi:10.1128/JB.00815-10 (2011). 
17 Steffes, C., Ellis, J., Wu, J. \& Rosen, B. P. The lysP gene encodes the lysine-specific permease. Journal of Bacteriology 174, 3242-3249 (1992).

18 Nguyen Le Minh, P. et al. Differential protein-DNA contacts for activation and repression by ArgP, a LysR-type (LTTR) transcriptional regulator in Escherichia coli. Microbiol Res 206, 141-158, doi:10.1016/j.micres.2017.10.009 (2018).

19 Bouvier, J., Stragier, P., Morales, V., Remy, E. \& Gutierrez, C. Lysine represses transcription of the Escherichia coli dapB gene by preventing its activation by the ArgP activator. Journal of Bacteriology 190, 5224-5229, doi:10.1128/JB.01782-07 (2008).

20 Marbaniang, C. N. \& Gowrishankar, J. Role of ArgP (IciA) in lysine-mediated repression in Escherichia coli. Journal of Bacteriology 193, 5985-5996, doi:10.1128/JB.05869-11 (2011).

21 Chaloupka, J. \& Strnadova, M. Turnover of murein in a diaminopimelic acid dependent mutant of Escherichia coli. Folia Microbiol (Praha) 17, 446-455, doi:10.1007/bf02872729 (1972).

22 Park, J. T. \& Uehara, T. How bacteria consume their own exoskeletons (turnover and recycling of cell wall peptidoglycan). Microbiol Mol Biol Rev 72, 211-227, table of contents, doi:10.1128/MMBR.00027-07 (2008).

23 Vollmer, W. Structural variation in the glycan strands of bacterial peptidoglycan. FEMS Microbiology Reviews 32, 287-306, doi:10.1111/j.1574-6976.2007.00088.x (2008).

24 Egan, A. J. \& Vollmer, W. The physiology of bacterial cell division. Annals of the New York Academy of Sciences 1277, 8-28, doi:10.1111/j.1749-6632.2012.06818.x (2013).

25 Linton, K. J. \& Higgins, C. F. The Escherichia coli ATP-binding cassette (ABC) proteins. Molecular Microbiology 28, 5-13, doi:10.1046/j.1365-2958.1998.00764.x (1998). 
26 Hogarth, B. G. \& Higgins, C. F. Genetic organization of the oligopeptide permease (opp) locus of Salmonella typhimurium and Escherichia coli. Journal of Bacteriology 153, 1548$1551(1983)$

27 Moussatova, A., Kandt, C., O'Mara, M. L. \& Tieleman, D. P. ATP-binding cassette transporters in Escherichia coli. Biochim Biophys Acta 1778, 1757-1771, doi:10.1016/j.bbamem.2008.06.009 (2008).

28 Higgins, C. F. \& Hardie, M. M. Periplasmic protein associated with the oligopeptide permeases of Salmonella typhimurium and Escherichia coli. Journal of Bacteriology 155, 1434-1438 (1983).

29 Higgins, C. F., Hardie, M. M., Jamieson, D. \& Powell, L. M. Genetic map of the opp (Oligopeptide permease) locus of Salmonella typhimurium. Journal of Bacteriology 153, 830-836 (1983).

30 Park, J. T., Raychaudhuri, D., Li, H., Normark, S. \& Mengin-Lecreulx, D. MppA, a periplasmic binding protein essential for import of the bacterial cell wall peptide L-alanylgamma-D-glutamyl-meso-diaminopimelate. Journal of Bacteriology 180, 1215-1223 (1998).

31 Bhatt, F., Patel, V. \& Jeffery, C. J. Open Conformation of the Escherichia coli Periplasmic Murein Tripeptide Binding Protein, MppA, at High Resolution. Biology (Basel) 7, doi:10.3390/biology7020030 (2018).

32 Maqbool, A. et al. Compensating stereochemical changes allow murein tripeptide to be accommodated in a conventional peptide-binding protein. The Journal of Biological Chemistry 286, 31512-31521, doi:10.1074/jbc.M111.267179 (2011). 
33 Maisonneuve, E. \& Gerdes, K. Molecular mechanisms underlying bacterial persisters. Cell 157, 539-548, doi:10.1016/j.cell.2014.02.050 (2014).

34 Lewis, K. Persister cells. Annual Review of Microbiology 64, 357-372, doi:10.1146/annurev.micro.112408.134306 (2010).

35 Keren, I., Kaldalu, N., Spoering, A., Wang, Y. \& Lewis, K. Persister cells and tolerance to antimicrobials. FEMS Microbiology Letters 230, 13-18, doi:10.1016/S03781097(03)00856-5 (2004).

36 Harms, A., Maisonneuve, E. \& Gerdes, K. Mechanisms of bacterial persistence during stress and antibiotic exposure. Science 354, doi:10.1126/science.aaf4268 (2016).

37 Jung, S. H., Ryu, C. M. \& Kim, J. S. Bacterial persistence: Fundamentals and clinical importance. J Microbiol 57, 829-835, doi:10.1007/s12275-019-9218-0 (2019).

38 Nikitushkin, V. D., Demina, G. R., Shleeva, M. O. \& Kaprelyants, A. S. Peptidoglycan fragments stimulate resuscitation of "non-culturable" mycobacteria. Antonie Van Leeuwenhoek 103, 37-46, doi:10.1007/s10482-012-9784-1 (2013).

39 Alteri, C. J., Smith, S. N. \& Mobley, H. L. Fitness of Escherichia coli during urinary tract infection requires gluconeogenesis and the TCA cycle. PLoS Pathogens 5, e1000448, doi:10.1371/journal.ppat.1000448 (2009).

40 Silverman, J. A., Schreiber, H. L. t., Hooton, T. M. \& Hultgren, S. J. From physiology to pharmacy: developments in the pathogenesis and treatment of recurrent urinary tract infections. Current Urology Reports 14, 448-456, doi:10.1007/s11934-013-0354-5 (2013).

41 Rosen, D. A., Hooton, T. M., Stamm, W. E., Humphrey, P. A. \& Hultgren, S. J. Detection of intracellular bacterial communities in human urinary tract infection. PLoS Medicine 4, e329, doi:10.1371/journal.pmed.0040329 (2007). 
42 Mysorekar, I. U. \& Hultgren, S. J. Mechanisms of uropathogenic Escherichia coli persistence and eradication from the urinary tract. Proceedings of the National Academy of Sciences of the United States of America 103, 14170-14175, doi:10.1073/pnas.0602136103 (2006).

43 Datsenko, K. A. \& Wanner, B. L. One-step inactivation of chromosomal genes in Escherichia coli K-12 using PCR products. Proceedings of the National Academy of Sciences of the United States of America 97, 6640-6645, doi:10.1073/pnas.120163297 (2000).

44 Desmarais, S. M., Cava, F., de Pedro, M. A. \& Huang, K. C. Isolation and preparation of bacterial cell walls for compositional analysis by ultra performance liquid chromatography. J Vis Exp, e51183, doi:10.3791/51183 (2014).

45 Blattner, F. R. et al. The complete genome sequence of Escherichia coli K-12. Science 277, 1453-1462 (1997).

46 Andersen, P. S. et al. Complete Genome Sequence of the Epidemic and Highly Virulent CTX-M-15-Producing H30-Rx Subclone of Escherichia coli ST131. Genome Announc 1, doi:10.1128/genomeA.00988-13 (2013).

47 Guzman, L. M., Belin, D., Carson, M. J. \& Beckwith, J. Tight regulation, modulation, and high-level expression by vectors containing the arabinose PBAD promoter. Journal of Bacteriology 177, 4121-4130 (1995). 
TABLE 1. Prevalence of quiescence among UPEC ST131 isolates

\begin{tabular}{|c|c|c|c|c|}
\hline Strain & Sequence type & Clade & $\begin{array}{c}\text { Quiescent at } 10^{3} \mathrm{CFU} \\
\text { and reversed by Lys, } \\
\text { Met and Tyr }{ }^{a}\end{array}$ & Source, reference \\
\hline CFT073 & ST73 & & + & $\underline{6}$ \\
\hline Nissle 1917 & ST73 & & + & $\underline{6}$ \\
\hline JJ1886 & ST131 & $\mathrm{C} 2$ & $+b$ & J. Johnson \\
\hline JJ1887 & ST131 & $\mathrm{C} 2$ & - & J. Johnson \\
\hline $\mathrm{JJ} 2547$ & ST131 & $\mathrm{C} 2$ & $+b$ & J. Johnson \\
\hline JJ2050 & ST131 & & - & J. Johnson \\
\hline $\mathrm{JJ} 2528$ & ST131 & $\mathrm{C} 1$ & - & J. Johnson \\
\hline MVAST0036 & ST131 & $\mathrm{C} 1$ & - & J. Johnson \\
\hline MVAST014 & ST131 & $\mathrm{C} 1$ & $+b$ & J. Johnson \\
\hline $\mathrm{H} 17$ & ST131 & $\mathrm{B} 0$ & - & J. Johnson \\
\hline JJ1901 & ST131 & & - & J. Johnson \\
\hline JJ2087 & ST131 & $\mathrm{B}$ & - & J. Johnson \\
\hline JJ1999 & ST131 & $\mathrm{B}$ & - & J. Johnson \\
\hline MVAST167 & ST131 & A & - & J. Johnson \\
\hline MVAST020 & ST131 & A & - & J. Johnson \\
\hline $\mathrm{JJ} 2134$ & ST131 & $\mathrm{C} 2$ & + & J. Johnson \\
\hline JJ2183 & ST131 & $\mathrm{C} 2$ & - & J. Johnson \\
\hline JJ2489 & ST131 & $\mathrm{C} 2$ & - & J. Johnson \\
\hline QUC02 & ST131 & & - & J. Johnson \\
\hline $\mathrm{JJ} 2118$ & ST131 & $\mathrm{C} 1$ & - & J. Johnson \\
\hline JJ2193 & ST131 & $\mathrm{C} 1$ & - & J. Johnson \\
\hline JJ2210 & ST131 & $\mathrm{C} 1$ & - & J. Johnson \\
\hline JJ2578 & ST131 & $\mathrm{C} 1$ & - & J. Johnson \\
\hline JJ2608 & ST131 & $\mathrm{C} 1$ & - & J. Johnson \\
\hline MVAST038 & ST131 & $\mathrm{C} 1$ & - & J. Johnson \\
\hline MVAST046 & ST131 & $\mathrm{C} 1$ & + & J. Johnson \\
\hline MVAST077 & ST131 & $\mathrm{C} 1$ & - & J. Johnson \\
\hline MVAST084 & ST131 & $\mathrm{C} 1$ & - & J. Johnson \\
\hline MVAST131 & ST131 & $\mathrm{C} 1$ & - & J. Johnson \\
\hline MVAST158 & ST131 & $\mathrm{C} 1$ & - & J. Johnson \\
\hline MVAST179 & ST131 & $\mathrm{C} 1$ & + & J. Johnson \\
\hline JJ2546 & ST131 & & - & E. Sokurenko \\
\hline JJ2548 & ST131 & & + & E. Sokurenko \\
\hline JJ2963 & ST131 & & - & E. Sokurenko \\
\hline JJ2974 & ST131 & & + & E. Sokurenko \\
\hline
\end{tabular}

${ }^{a}$ Represents strains seeded $\leq 10^{3} \mathrm{CFU}$ in $0.2 \%$ glucose M9 minimal media $37^{\circ} \mathrm{C} 24 \mathrm{~h},(+)$ represents quiescent, (-) represents non-quiescent.

${ }^{b}$ Denotes strains that are non-quiescent when seeded at $10^{5} \mathrm{CFU}$ on $0.2 \%$ glucose M9 minimal media and grown at $37^{\circ} \mathrm{C}$ for $24 \mathrm{~h}$. 


\section{Figure Legends}

Fig. 1. The quorum-regulated quiescent state of $E$. coli CFT073 cells on glucose M9 minimal agar is reversible. (A) CFT073 cells $\left(10^{4}\right)$ were plated onto M9 minimal agar with $0.2 \%$ glucose. A $5 \mu 1$ solution of L-lysine $(1 \mathrm{mM})$ and L-methionine $(1 \mathrm{mM})$, sterile human urine, or an actively growing colony of CFT073 was transferred to the center of the plate, cells were incubated for $24 \mathrm{~h}$, and the zone of growth surrounding the challenge site was monitored. $(B, C)$ Zones of growth expand with time after addition of an actively growing colony of CFT073 up to $72 \mathrm{~h}$. (D) Removal of the actively growing colony at $24 \mathrm{~h}$ has no effect on zonal expansion. White arrows indicate position of stimulant addition, brackets indicate the zone of colony growth and scale bars are $1 \mathrm{~cm}$ in length.

Fig. 2. Quiescent CFT073 cells are filamentous at 24 h. (A) CFT073 cells were grown on LB agar or $0.2 \%$ glucose minimal agar with and without L-lysine $(1 \mathrm{mM})$ and L-methionine $(1 \mathrm{mM})$, harvested at $24 \mathrm{~h}$ and visualized by DIC microscopy. Scale bars are $5 \mu \mathrm{m}$ in length. (B) Violin plot representing cell length distribution at $24 \mathrm{~h}$ for 200 cells grown under conditions described in (A).

\section{Fig. 3. Quiescent CFT073 $\Delta y s P$ and $\Delta a r g P$ cells fail to proliferate with L-lysine}

CFT073, CFT073 $\Delta l y s P$, CFT073 $\Delta l y s P$ with pLysP, CFT073 $\Delta l y s P$ with pBad24, CFT073 $\triangle \arg P, \mathrm{CFT} 073 \Delta \arg P$ with pArgP and CFT073 $\Delta \operatorname{argP}$ with pBad24 cells $\left(10^{4}\right)$ were plated onto M9 minimal agar with $0.2 \%$ glucose. All vector carrying strains were plated onto agar containing $50 \mu \mathrm{g} / \mathrm{ml}$ Ampicillin, while CFT073 $\Delta l y s P$ with pLysP and CFT073 $\Delta l y s P$ with 
pBad 24 were plated onto agar with $0.1 \%$ arabinose. Quiescent cells were challenged with a 5 $\mu l$ solution of L-lysine $(0.5 \mathrm{mM})$ and L-methionine $(1 \mathrm{mM})($ CFT073 $\Delta l y s P$, CFT073 $\Delta l y s P$ with pLysP, CFT073 $\Delta l y s P$ with pBad24), L-lysine $(1 \mathrm{mM})$ and L-methionine $(1 \mathrm{mM})(\mathrm{CFT}$ $\triangle \operatorname{argP}, \mathrm{CFT} 073 \triangle \arg P$ with $\mathrm{pArgP}$ and CFT073 $\triangle \operatorname{argP}$ with $\mathrm{pBad} 24)$, sterile human urine or an actively growing colony of MG1655 transferred to the center of the plate and cells were incubated for $24 \mathrm{~h}$.

\section{Fig. 4. ST131 strain JJ1886 displays quorum dependent growth}

(A) JJ1886 cells were plated onto M9 minimal agar with $0.2 \%$ glucose and LB agar, at $10^{5}$ and $10^{3} \mathrm{CFU}$. JJ1886 displays quorum dependent growth at $10^{3} \mathrm{CFU}$ on glucose M9 minimal agar. (B) JJ1886 cells $\left(10^{3}\right)$ were plated onto M9 minimal agar with $0.2 \%$ glucose. A $5 \mu$ l solution of L-lysine (1 mM) and L-methionine (1 mM), L-lysine (1 mM), L-methionine (1 mM), sterile human urine, or an actively growing colony of CFT073 was transferred onto a JJ1886 seeded plate and cells were incubated for $24 \mathrm{~h}$.

\section{Fig. 5. Peptidoglycan fragments promote proliferation of CFT073 and JJ1886 cells}

LCMS profiles of total ion counts (TIC) including (A) mutanolysin digested crude PG, which was tested for stimulating proliferation of quiescent JJ1886 cells plated at $10^{3} \mathrm{CFU}$ on M9 minimal agar with $0.2 \%$ glucose, inset; (B) elution of a PG tripeptide similar to an E. coli fragment and corresponding test for quiescence reversal, inset; (C) elution of a PG pentapeptide similar to a $S$. aureus fragment and corresponding test for quiescence reversal, inset; (D) elution of a NAG-NAM disaccharide and corresponding test for quiescence reversal, inset; and (E) elution of a disaccharide tetrapeptide isolated from the mutanolysin-digested 
crude PG corresponding test for quiescence reversal, inset. Candidate proliferants were collected tested in the quiescence assay as described in Material and Methods and plates were incubated at $37^{\circ} \mathrm{C}$ for $24 \mathrm{~h}$. White arrows indicate position of proliferant addition and scale bar is $1 \mathrm{~cm}$ in length. 
bioRxiv preprint doi: https://doi.org/10.1101/830877; this version posted November 4, 2019. The copyright holder for this preprint (which was not certified by peer review) is the author/funder, who has granted bioRxiv a license to display the preprint in perpetuity. It is made available under aCC-BY-NC-ND 4.0 International license.

Fig. 1

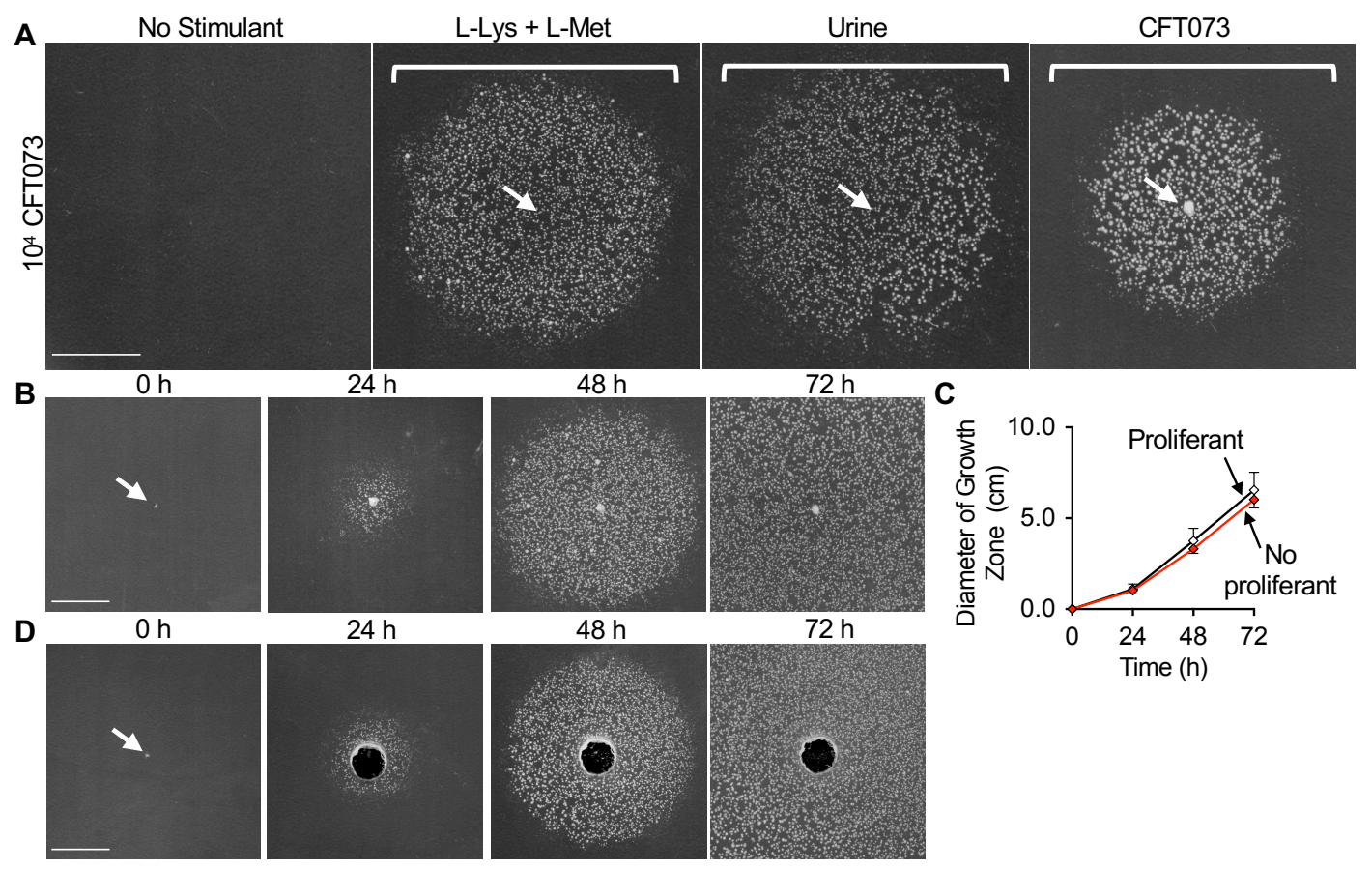


bioRxiv preprint doi: https://doi.org/10.1101/830877; this version posted November 4, 2019. The copyright holder for this preprint (which was not certified by peer review) is the author/funder, who has granted bioRxiv a license to display the preprint in perpetuity. It is made available under aCC-BY-NC-ND 4.0 International license.

Fig. 2
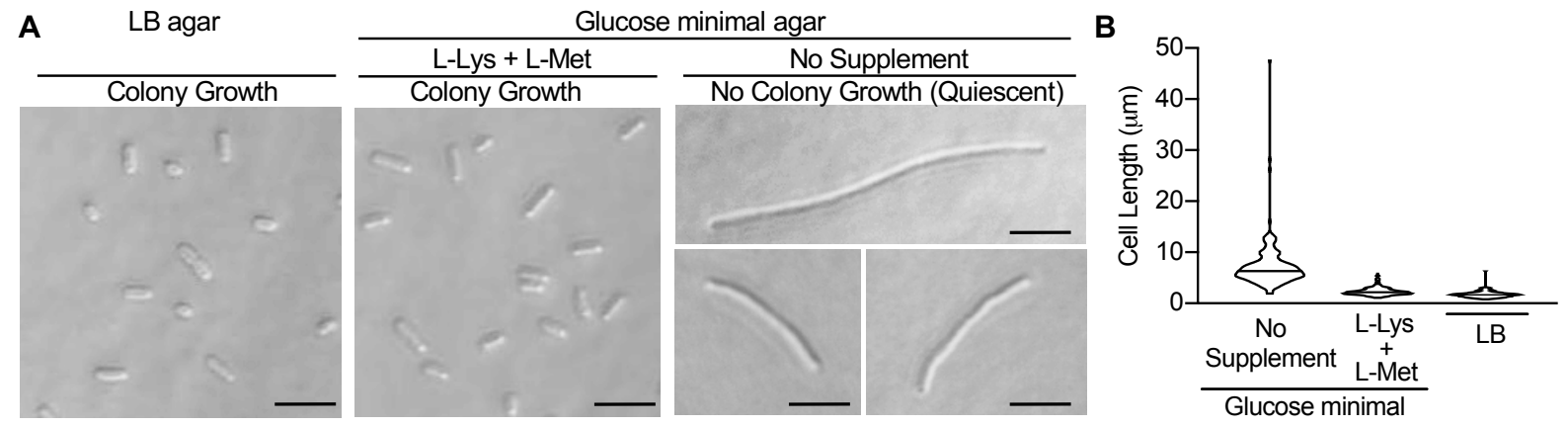
bioRxiv preprint doi: https://doi.org/10.1101/830877; this version posted November 4, 2019. The copyright holder for this preprint (which was not certified by peer review) is the author/funder, who has granted bioRxiv a license to display the preprint in perpetuity. It is made available under aCC-BY-NC-ND 4.0 International license.

Fig. 3
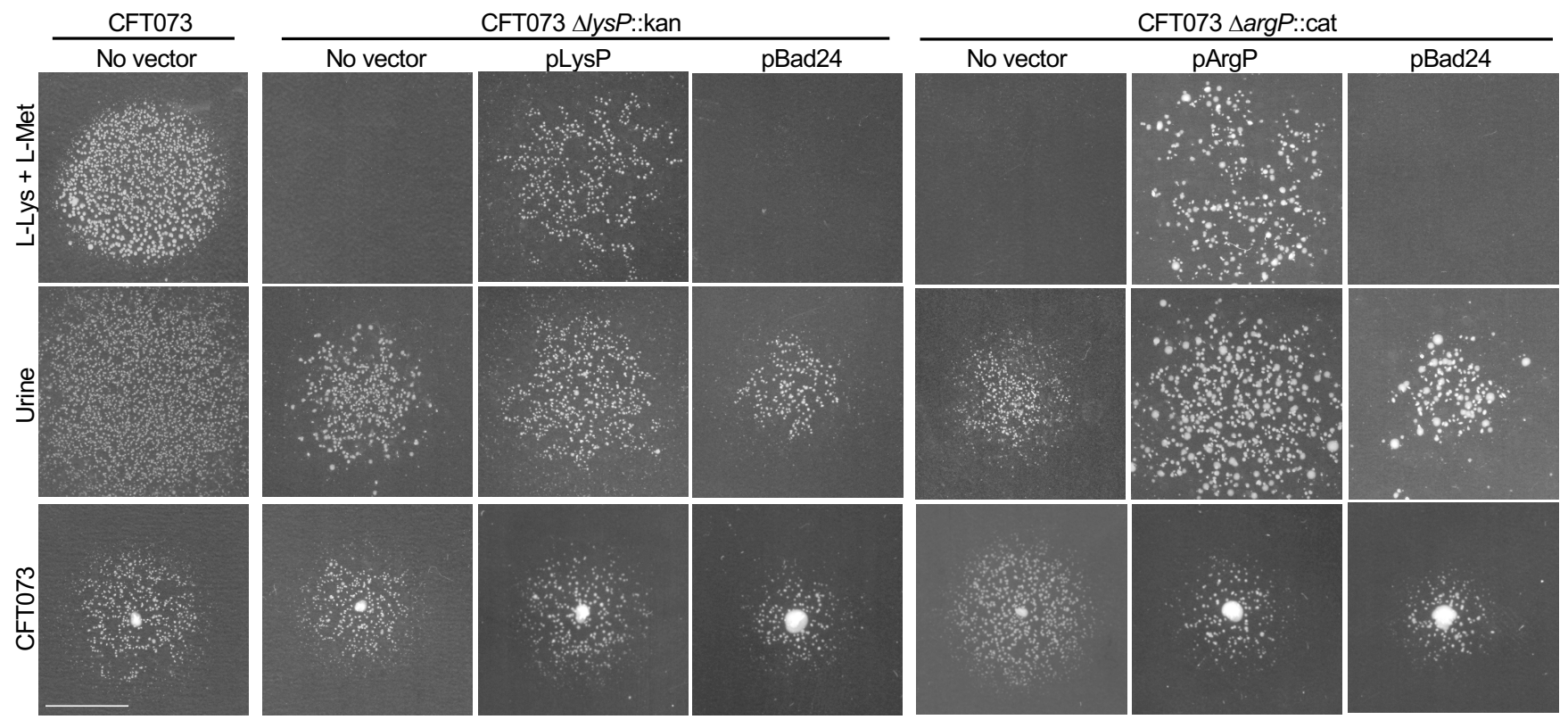
bioRxiv preprint doi: https://doi.org/10.1101/830877; this version posted November 4,2019 . The copyright holder for this preprint (which was not certified by peer review) is the author/funder, who has granted bioRxiv a license to display the preprint in perpetuity. It is made available under aCC-BY-NC-ND 4.0 International license.

Fig. 4

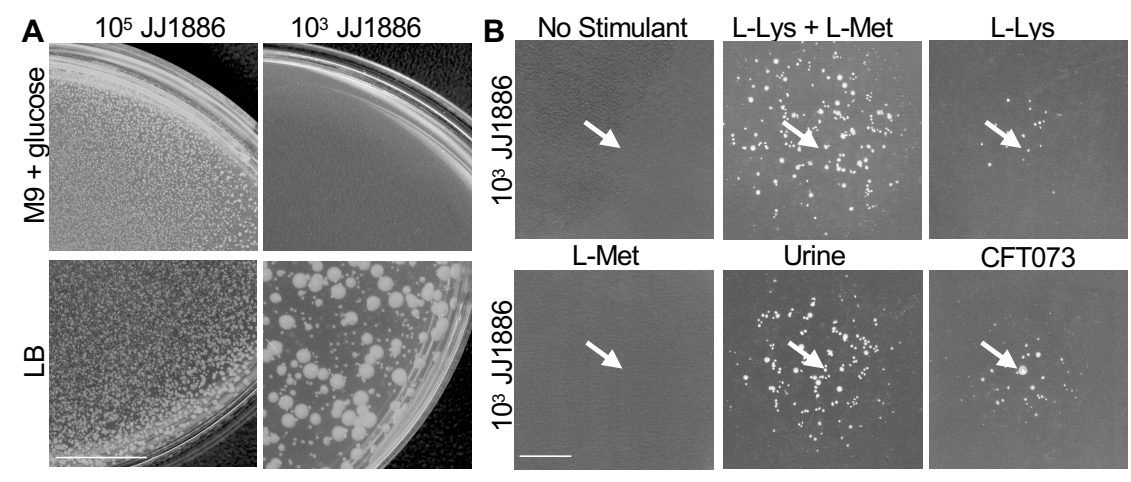


Fig. 5
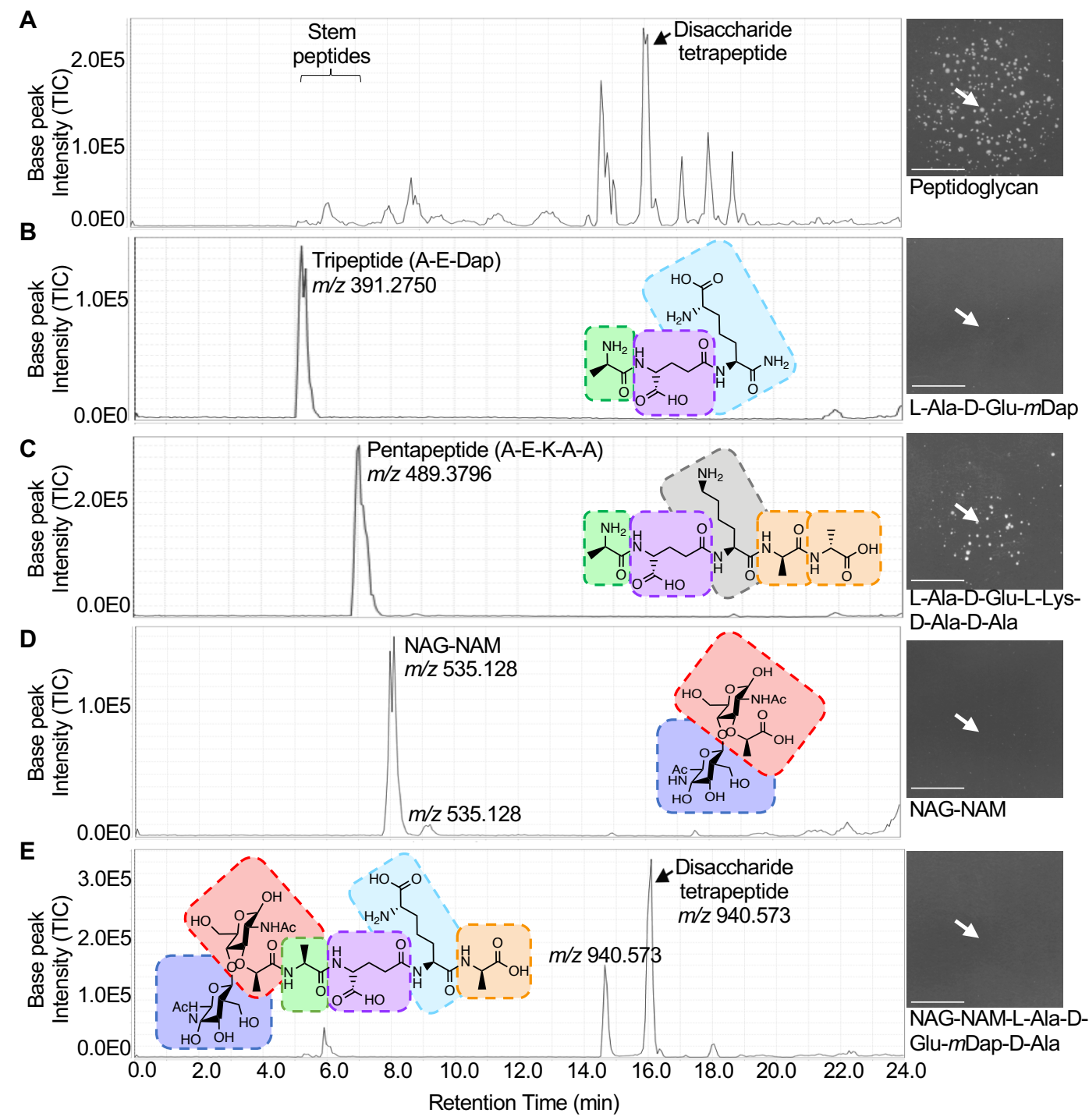